\title{
Compute-and-Forward on Wireless Lattice Networks with Local Interference
}

\author{
Jasper Goseling ${ }^{* \dagger}$, Jos H. Weber ${ }^{\dagger}$ and Michael Gastpar ${ }^{\ddagger \dagger}$ \\ * Stochastic Operations Research, \\ University of Twente, The Netherlands \\ $\dagger$ Delft University of Technology, The Netherlands \\ $\ddagger$ Laboratory for Information in Networked Systems, \\ Ecole Polytechnique Fédérale de Lausanne, Switzerland \\ j.goseling@utwente.nl, j.h.weber@tudelft.nl, michael.gastpar@epfl.ch
}

\begin{abstract}
The capacity of a wireless lattice network with local interference is studied. In particular, the transport capacity under a multiple unicast traffic pattern is studied for a network of nodes placed on a hexagonal lattice. Different ways of handling broadcast and superposition lead to various communication models. In particular we consider models arising from the use of compute-and-forward/physical layer network coding.

If neither broadcast nor superposition is exploited, the normalized transport capacity was previously shown to be between $1 / 3$ and $2 / 5$. In this work it is shown that exploiting either broadcast or superposition increases the capacity to at least $3 / 7$, hence providing a strict improvement.
\end{abstract}

\section{INTRODUCTION}

The classical means of operating a wireless network tries to avoid interference and basically provides an abstraction of the network as a set of reliable point-to-point bit pipes. Compute-and-forward [1] makes use of the interference and allows nodes in a wireless network to reliably obtain linear combinations of messages transmitted by others in the network as well as to broadcast messages to multiple nodes. We study the resulting abstraction of the network, which is no longer a set of point-to-point links. In particular we study the impact of compute-and-forward on the transport capacity of wireless networks.

We separate the effects of interference into broadcast and superposition and study the impact on capacity of exploiting each of these individually. In particular, we consider four different communication models, that capture the abstractions of the wireless medium resulting from different ways of handling broadcast and superposition.

The four models, introduced in [2] are denoted by $P / P, B / P$, $P / M$ and $B / M$. The first position denotes whether symbols are transmitted to a single neighbour $(P)$ or broadcast to all neighbours (B). The second position denotes whether multiple transmissions to a node cause interference $(P)$ or that nodes receive the sum of all symbols that are transmitted by neighbours (M). More precisely, we have

1) $P / P$ : Neither broadcast nor superposition is exploited, i.e., a single transmission can be received by at most one device and multiple transmissions to the same device result in a collision.
2) B/P: Transmissions are received by all neighbours. However, multiple transmissions to the same device lead to a collision.

3) $P / M$ : Nodes can decode the sum of all transmissions by neighbouring nodes. However, a single transmission can be received by at most one device.

4) B/M: A combination of the above two effects into a model that involves both the broadcast and the superposition effects.

We study the transport capacity of a network in which 1) nodes are located on the hexagonal lattice and 2) there is only local interference. Previous results provided lower and upper bounds on the transport capacity for all four models [2]. The contribution of the current work consists of improved lower bounds for the $\mathrm{B} / \mathrm{P}$ and $\mathrm{P} / \mathrm{M}$ models. These new lower bounds are strictly larger than the upper bound for $P / P$, hence exploiting broadcast or interference improves transport capacity. Our previous bounds [2] are not tight enough to draw this conclusion.

The method used to obtain the improved lower bounds is to carefully construct a set of unicast sessions. For this set of unicast sessions we construct a network code. This code has the properties that 1) no intermediate node in the network can recover the source symbols it is receiving in linear combinations (as would be required in the COPE framework [3], for instance), 2) source symbols are only retransmitted in linear combinations by nodes that are on the shortest path between source and destination, 3) destination nodes can decode the required messages.

Note, finally, that our focus is not on designing the channel codes that are required for compute-and-forward. We assume that these are given and analyze the implications in large networks. For an overview of results on channel coding for compute-and-forward [1] and the related notions of physicallayer/analog network coding [4], [5], see [6].

The outline of the remainder of this paper is as follows. In Section II we give an exact formulation of the model and problem statement. Section III provides an overview of the results. The lower bounds for the $\mathrm{P} / \mathrm{M}$ and $\mathrm{B} / \mathrm{P}$ models are given in Sections IV and $\mathrm{V}$ respectively. 


\section{MODEL}

\section{A. Network topology}

We consider a network of size $(K+2) \times(K+2)$, with nodes located on the hexagonal lattice and edges between nearest neighbours. We index nodes with a vector $u \in \mathbb{N}^{2}$, where we write $u=(u[1], u[2])$. The location in $\mathbb{R}^{2}$ of $u$ is $u G_{\Lambda}$, with $G_{\Lambda}=\left[\begin{array}{cc}1 & 0 \\ 1 / 2 & \sqrt{3} / 2\end{array}\right]$. Now, we consider $(V, E)$ with

$V=\left\{u \in \mathbb{N}^{2} \mid 0 \leq u[1] \leq K+1,0 \leq u[2] \leq K+1\right\}$,

$E=\left\{(u, v) \subset V \times V \mid\left\|(u[1]-v[1], u[2]-v[2]) G_{\Lambda}\right\|_{2}=1\right\}$

Let $e_{1}=(1,0)$ and $e_{2}=(0,1)$.

Let

$$
\begin{aligned}
L & =\{u \in V \mid u[1]=0,1 \leq u[2] \leq K\}, \\
R & =\{u \in V \mid u[1]=K+1,1 \leq u[2] \leq K\}, \\
T & =\{u \in V \mid 1 \leq u[1] \leq K, u[2]=K+1\}, \\
B & =\{u \in V \mid 1 \leq u[1] \leq K, u[2]=0\}, \\
I & =\{u \in V \mid 1 \leq u[1] \leq K, 1 \leq u[2] \leq K\},
\end{aligned}
$$

i.e., we have left, right, top and bottom boundary of $V$ and its interior. Let $C=L \cup R \cup T \cup B$. Finally, we will denote by $u_{\mathrm{l}} \in L, u_{\mathrm{r}} \in R, u_{\mathrm{t}} \in T, u_{\mathrm{b}} \in B$ and $u_{\mathrm{i}} \in I$, arbitrary nodes in these sets.

\section{B. Communication Models}

Time is slotted. Symbols are from the finite field $\mathbb{F}_{2}$, i.e., we consider bits. In all four models each link can carry one bit per time slot, i.e., links have unit capacity. Let $\tilde{X}_{n}(u)$ and $\tilde{Y}_{n}(u)$ be the symbols transmitted and received respectively, by node $u$ in time slot $n$. Let $N_{u}=\{v \in V \mid(v, u) \in E\}$ denote the neighbourhood of $u$. The channel output $\tilde{Y}_{n}(v)$ depends only on the channel inputs of neighbouring nodes in the same time slot. All our models respect half-duplex constraints, meaning that no node can simultaneously transmit and receive.

The functional relation between channel inputs and outputs is now specified for the $\mathrm{P} / \mathrm{M}$ and $\mathrm{B} / \mathrm{P}$ models. To simplify notation for the $\mathrm{P} / \mathrm{M}$ model, we introduce variables $A(u), u \in$ $V$, that denote the neighbour that $u$ is transmitting to. The models, as defined in [2], are as follows.

$\mathrm{P} / \mathrm{M}$ : Superposition is exploited, but broadcast is not, i.e., $\tilde{Y}_{n}(u)=\sum_{v \in N_{u}: A(v)=u} \tilde{X}_{n}(v)$, iff all nodes in $u \cup$ $\left\{v \in N_{u}: A(v) \neq u\right\}$ remain silent.

$\mathrm{B} / \mathrm{P}:$ Broadcast is exploited, but superposition is not, i.e., $\tilde{Y}_{n}(u)=\tilde{X}_{n}(v)$, iff $u \in N_{v}$ and all nodes in $u \cup$ $N_{u} \backslash\{v\}$ remain silent.

If half-duplex or interference constraints are not satisfied, the channel output is uniformly distributed and no information is obtained about the channel inputs, see [2] for details.

\section{Transport Capacity}

The traffic pattern that we consider is multiple unicast. For a set of $M$ unicast sessions, let $s(m)$ and $r(m)$ denote the source and destination, respectively, of the $m$ th session, and $\mathcal{R}(m)$ its throughput. Our measure of interest is the

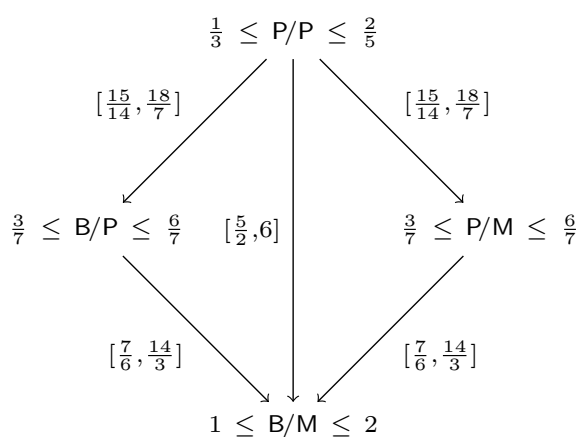

Fig. 1. Transport capacity of the hexagonal lattice under different models For each model lower and upper bounds on transport capacity are presented. Labels next to arrows denote the range (lower and upper bounds) of the multiplicative improvement obtained by moving from one model to the next.

transport capacity of a network which is defined as the maximum, over all $M$, all configurations of unicast sessions on a given network and all possible transmission strategies, of $\sum_{m=1}^{M} \operatorname{dist}(s(m), r(m)) \mathcal{R}(m) /|V|$, where $\operatorname{dist}(s(m), r(m))$ is the number of hops on the shortest path from $s(m)$ to $r(m)$. The transport capacity is the maximum number of bits $x$ hops per time slot, normalized by network size, that can be transported in the network. Note that the transport capacity can be interpreted as a projection of part of the boundary of the (high-dimensional) capacity region of the network.

Our interest is in the transport capacity of the hexagonal lattice in the limit of large number of nodes.

\section{Notation}

If $f(x)=\mathrm{o}(g(x))$, then $\lim _{x \rightarrow \infty} f(x) / g(x)=0$. For integers $a, b$ and $p>0, a \equiv b(\bmod p)$ iff $a-b$ is divisible by $p$.

\section{RESULTS}

In this work we present lower bounds on the transport capacity of the hexagonal lattice under the $P / M$ and $B / P$ models. These bounds provide an improvement over earlier bounds given in [2].

Theorem 1. The transport capacity of the hexagonal lattice under the $\mathrm{P} / \mathrm{M}$ and $\mathrm{B} / \mathrm{P}$ models is at least $3 / 7$.

Upper bounds for all models and lower bounds for the $P / P$ and $\mathrm{B} / \mathrm{M}$ models are given in [2]. An overview of the best known lower and upper bounds for all models is given in Figure 1. The previous lower bounds for the $P / M$ and $B / P$ models are $2 / 5$. Note that this corresponds to the best known upper bound for the $\mathrm{P} / \mathrm{P}$ model. Hence, previously, it was not possible to claim that exploiting superposition or broadcast would improve transport capacity.

\section{Achievable Strategy under P/M}

The strategy is based on the network codes developed in [7]. An outline of the strategy is as follows: 


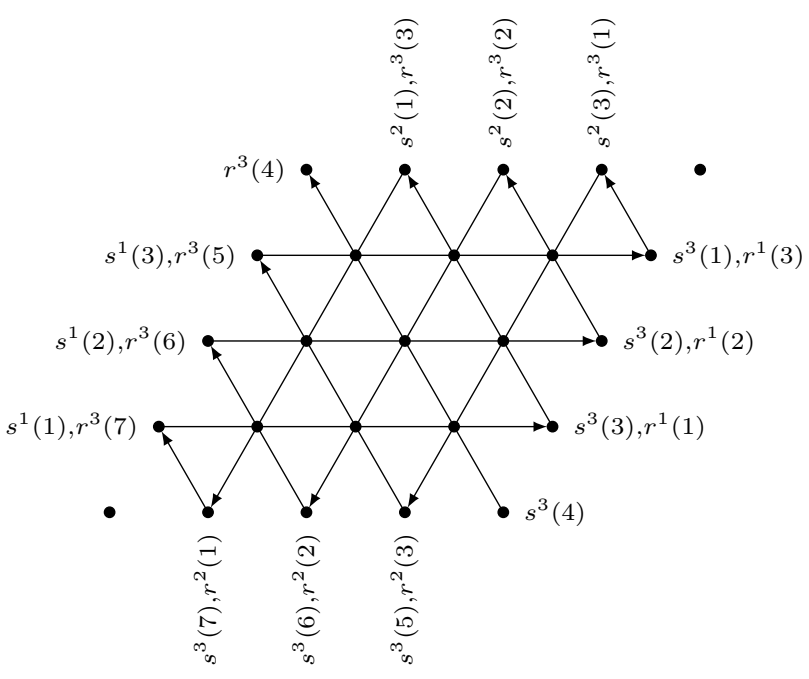

Fig. 2. Sessions for the $\mathrm{P} / \mathrm{M}$ strategy for $K=3$.

1) Construct $4 K+1$ sessions, such that total length of all sessions is $3 K(K+1)$.

2) Operate in rounds of 7 time slots.

3) In each round deliver, for each session, one symbol at each destination.

The different elements of the strategy are covered in subsequent subsections.

\section{A. Sessions}

Remember that our interest is in the transport capacity, which is defined in terms of a maximum over all possible multiple unicast configurations. Therefore we can carefully place unicast session and analyze the resulting performance.

In the current work we construct $4 K+1$ unicast sessions. These sessions are denoted by $m^{1}(i), m^{2}(j)$ and $m^{3}(k)$, where $1 \leq i \leq K, 1 \leq j \leq K$ and $1 \leq k \leq 2 K+1$. Session $m^{\bar{d}}(i), \bar{d} \in\{1,2,3\}$, has the sequence of message symbols $m_{0}^{d}(i), m_{1}^{d}(i), m_{2}^{d}(i), \ldots$ to be transferred. In the achievable strategy session $m^{3}(K+1)$ will be given throughput zero. Hence, we put all its message symbols to zero, $m_{t}^{3}(K+1)=0$ for all $t$.

The source and destination of session $m^{d}(i)$ are denoted by $s^{d}(i)$ and $r^{d}(i)$ respectively. Sources and destinations are positioned, as depicted in Figure 2, i.e., as

$$
\begin{aligned}
s^{1}(i)= & (0, i), \quad r^{1}(i)=(K+1, i), \\
s^{2}(j)= & (j, K+1), \quad r^{2}(j)=(j, 0), \\
s^{3}(k)= & (K+1+\min \{0, K+1-k\}, \\
& \max \{0, K+1-k\}), \\
r^{3}(k)= & (\max \{0, K+1-k\}, \\
& K+1+\min \{0, K+1-k\}) .
\end{aligned}
$$

Let $\lambda^{d}(i)$ denote the number of hops on the shortest path between the source and destination of session $m^{d}(i)$. We have $\lambda^{1}(i)=\lambda^{2}(i)=K+1$ and

$$
\sum_{k=1}^{K} \lambda^{3}(k)+\sum_{k=K+2}^{2 K+1} \lambda^{3}(k)=2 \sum_{k=1}^{K} k=K(K+1) .
$$

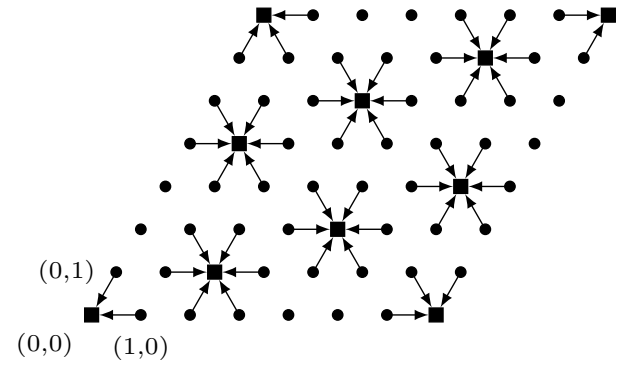

Fig. 3. Scheduling for $\mathrm{P} / \mathrm{M}$ in 0 -th time slot of a round. Square nodes denote $A_{0}$.

Therefore, the total number of hops, excluding $m^{3}(K+1)$, is $3 K(K+1)$.

For notational convenience later on, we introduce the functions $\kappa^{1}: V \rightarrow\{1, \ldots, K\}, \kappa^{2}: V \rightarrow\{1, \ldots, K\}$, $\kappa^{3}: V \rightarrow\{1, \ldots, 2 K+1\}$ that map a node in $V$ to the index of the session that has $u$ on its shortest path between source and destination. More precisely we have

$$
\begin{aligned}
& \kappa^{1}(u)=u[2], \\
& \kappa^{2}(u)=u[1], \\
& \kappa^{3}(u)=2 K+2-u[1]-u[2] .
\end{aligned}
$$

Moreover we will use the functions $\delta^{1}: V \rightarrow\{0,1, \ldots\}$, $\delta^{2}: V \rightarrow\{0,1, \ldots\}, \delta^{3}: V \rightarrow\{0,1, \ldots\}$ that map a node to the number of hops from $u$ to $s^{1}\left(\kappa^{1}(u)\right), s^{2}\left(\kappa^{2}(u)\right)$ and $s^{3}\left(\kappa^{3}(u)\right)$ respectively. More precisely we have

$$
\begin{aligned}
& \delta^{1}(u)=u[1], \\
& \delta^{2}(u)=K+1-u[2], \\
& \delta^{3}(u)= \begin{cases}u[2], & \text { if } u[1]+u[2] \leq K+1, \\
K+1-u[1], & \text { if } u[1]+u[2]>K+1 .\end{cases}
\end{aligned}
$$

\section{B. Scheduling}

We use rounds of 7 time slots, counting time slots in a round from 0 to 6 . Scheduling in the $i$-th time slot of a round is based on the set of nodes

$$
A_{i}=\{u \in V \mid i \equiv u[1]-2 u[2](\bmod 7)\} .
$$

Now from $A_{i}$ we construct the set $B_{i}$ defined as

$$
B_{i}=\left\{(u, v) \in V \times V \mid(u, v) \in E, v \in A_{i}\right\} .
$$

If $(u, v) \in B_{i}$, then in time slot $i$ node $u$ transmits to node $v$. The schedule is illustrated in Figure 3.

It is readily verified that for any node $u \in V$ :

1) In each round $u$ is scheduled to transmit to each of its neighbours once. If $u \in C$ it will not use all its scheduled slots.

2) In the remaining time slot that $u$ is not transmitting it is receiving. In that time slot all neighbours of $u$ are scheduled to transmit to $u$.

We denote by $x_{t}(u, v)$ the symbol transmitted by $u$ to $v$ in round $t$ and by $y_{t}(u)$ the symbol received by $u$ in round $t$. 


\section{Network code}

In this section we present the network code that is employed. We present details only for nodes in $I$ and $L$, in part due to space constraints, but also to avoid repetition.

The network code is such that nodes in the interior transmit as follows:

$$
\begin{aligned}
x_{t}\left(u_{\mathrm{i}}, u_{\mathrm{i}}-e_{1}\right) & =y_{t-2}\left(u_{\mathrm{i}}\right)+x_{t-2}\left(u_{\mathrm{i}}, u_{\mathrm{i}}-e_{1}\right), \\
x_{t}\left(u_{\mathrm{i}}, u_{\mathrm{i}}-e_{1}+e_{2}\right) & =y_{t-1}\left(u_{\mathrm{i}}\right)+x_{t-1}\left(u_{\mathrm{i}}, u_{\mathrm{i}}-e_{1}+e_{2}\right), \\
x_{t}\left(u_{\mathrm{i}}, u_{\mathrm{i}}+e_{2}\right) & =y_{t-2}\left(u_{\mathrm{i}}\right)+x_{t-2}\left(u_{\mathrm{i}}, u_{\mathrm{i}}+e_{2}\right), \\
x_{t}\left(u_{\mathrm{i}}, u_{\mathrm{i}}+e_{1}\right) & =y_{t-1}\left(u_{\mathrm{i}}\right)+x_{t-1}\left(u_{\mathrm{i}}, u_{\mathrm{i}}+e_{1}\right), \\
x_{t}\left(u_{\mathrm{i}}, u_{\mathrm{i}}+e_{1}-e_{2}\right) & =y_{t-2}\left(u_{\mathrm{i}}\right)+x_{t-2}\left(u_{\mathrm{i}}, u_{\mathrm{i}}+e_{1}-e_{2}\right), \\
x_{t}\left(u_{\mathrm{i}}, u_{\mathrm{i}}-e_{2}\right) & =y_{t-1}\left(u_{\mathrm{i}}\right)+x_{t-1}\left(u_{\mathrm{i}}, u_{\mathrm{i}}-e_{2}\right)
\end{aligned}
$$

and for nodes on the left border we have

$$
\begin{aligned}
x_{t}\left(u_{1}, u_{1}+e_{2}\right)= & m_{t-1}^{1}\left(\kappa^{1}\left(u_{1}\right)\right), \\
x_{t}\left(u_{1}, u_{1}+e_{1}\right)= & y_{t-1}\left(u_{1}\right)+ \\
& m_{t}^{1}\left(\kappa^{1}\left(u_{1}\right)\right)+m_{t-2}^{1}\left(\kappa^{1}\left(u_{1}\right)\right), \\
x_{t}\left(u_{1}, u_{1}+e_{1}-e_{2}\right)= & y_{t-2}\left(u_{1}\right)+ \\
& m_{t-1}^{1}\left(\kappa^{1}\left(u_{1}\right)\right)+m_{t-3}^{1}\left(\kappa^{1}\left(u_{1}\right)\right), \\
x_{t}\left(u_{1}, u_{1}-e_{2}\right)= & y_{t-1}\left(u_{1}\right)+m_{t-2}^{1}\left(\kappa^{1}\left(u_{1}\right)\right) .
\end{aligned}
$$

It follows, as stated in the following lemma, that by using the code described above, message symbols are only retransmitted by nodes that are on the shortest path between source and destination.

Lemma 1. Let $u_{\mathrm{i}} \in I, u_{\mathrm{l}} \in L$. For all $t \geq 0$, the transmitted and received signals at these nodes satisfy

$$
\begin{aligned}
y_{t}\left(u_{\mathrm{i}}\right)= & \sum_{d=1}^{3}\left(m_{t+1-\delta^{d}\left(u_{\mathrm{i}}\right)}^{d}\left(\kappa^{d}\left(u_{\mathrm{i}}\right)\right)\right. \\
& \left.+m_{t-1-\delta^{d}\left(u_{\mathrm{i}}\right)}^{d}\left(\kappa^{d}\left(u_{\mathrm{i}}\right)\right)\right), \\
y_{t}\left(u_{\mathrm{l}}\right)= & m_{t-1}^{1}\left(\kappa^{1}\left(u_{1}\right)\right)+m_{t+1-\delta^{3}\left(u_{1}\right)}^{3}\left(\kappa^{3}\left(u_{1}\right)\right), \\
x_{t}\left(u_{1}, u_{1}+e_{2}\right)= & m_{t-1}^{1}\left(\kappa^{1}\left(u_{1}\right)\right), \\
x_{t}\left(u_{1}, u_{1}+e_{1}\right)= & m_{t}^{1}\left(\kappa^{1}\left(u_{1}\right)\right)+m_{t-\delta^{3}\left(u_{1}\right)}^{3}\left(\kappa^{3}\left(u_{1}\right)\right), \\
x_{t}\left(u_{1}, u_{1}+e_{1}-e_{2}\right)= & m_{t-1}^{1}\left(\kappa^{1}\left(u_{1}\right)\right)+m_{t-1-\delta^{3}\left(u_{1}\right)}^{3}\left(\kappa^{3}\left(u_{1}\right)\right), \\
x_{t}\left(u_{1}, u_{1}-e_{2}\right)= & m_{t-\delta^{3}\left(u_{1}\right)}^{3}\left(\kappa^{3}\left(u_{1}\right)\right), \\
x_{t}\left(u_{\mathrm{i}}, w\right)= & \sum_{d=1}^{3} m_{t-\tau-\delta^{d}\left(u_{\mathrm{i}}\right)}^{d}\left(\kappa^{d}\left(u_{\mathrm{i}}\right)\right),
\end{aligned}
$$

where $\tau=0$ if $w \in\left\{u_{\mathrm{i}}+e_{1}, u_{\mathrm{i}}-e_{1}+e_{2}, u_{\mathrm{i}}-e_{2}\right\}$ and $\tau=1$ otherwise.

Proof: We use induction over $t$. At $t=0$ the conditions are obviously satisfied. Now suppose that the lemma holds for all rounds before $t$. The induction step is tedious, but straightforward and will be proven only for $y_{t}(u), u \in L$, with $1<u[2]<K$. The last condition ensures that $u+e_{2} \in L$, $u-e_{2} \in L$ and $u+e_{1}-e_{2} \in I$. At round $t$, by using the coding rules and the induction hypothesis, we have

$$
\begin{aligned}
y_{t}(u)= & x_{t}\left(u+e_{2}, u\right)+x_{t}\left(u+e_{1}, u\right) \\
& +x_{t}\left(u+e_{1}-e_{2}, u\right)+x_{t}\left(u-e_{2}, u\right) \\
= & m_{t-\delta^{3}\left(u+e_{2}\right)}^{3}\left(\kappa\left(u+e_{2}\right)\right) \\
& +\sum_{d=1}^{3} m_{t-1-\delta^{d}\left(u+e_{1}\right)}^{d}\left(\kappa^{d}\left(u+e_{1}\right)\right) \\
& +\sum_{d=1}^{3} m_{t-\delta^{d}\left(u+e_{1}-e_{2}\right)}^{d}\left(\kappa^{d}\left(u+e_{1}-e_{2}\right)\right) \\
& +m_{t-1-\delta^{1}\left(u-e_{2}\right)}^{1}\left(\kappa\left(u-e_{2}\right)\right) .
\end{aligned}
$$

Now, from $\delta^{1}\left(u-e_{2}\right)=1+\delta^{1}\left(u+e_{1}-e_{2}\right)$ and $\kappa^{1}\left(u-e_{2}\right)=$ $\kappa^{1}\left(u+e_{1}-e_{2}\right)$ it follows that

$$
\begin{aligned}
& m_{t-1-\delta^{1}\left(u-e_{2}\right)}^{1}\left(\kappa^{1}\left(u-e_{2}\right)\right)= \\
& m_{t-\delta^{1}\left(u+e_{1}-e_{2}\right)}^{2}\left(\kappa^{1}\left(u+e_{1}-e_{2}\right)\right) .
\end{aligned}
$$

Similarly

$$
\begin{aligned}
& m_{t-1-\delta^{2}\left(u+e_{1}\right)}^{2}\left(\kappa^{2}\left(u+e_{1}\right)\right)= \\
& m_{t-\delta^{2}\left(u+e_{1}-e_{2}\right)}^{2}\left(\kappa^{2}\left(u+e_{1}-e_{2}\right)\right),
\end{aligned}
$$

and

$$
m_{t-\delta^{3}\left(u+e_{2}\right)}^{3}\left(\kappa\left(u+e_{2}\right)\right)=m_{t-1-\delta^{3}\left(u+e_{1}\right)}^{3}\left(\kappa^{3}\left(u+e_{1}\right)\right) .
$$

Hence

$$
\begin{aligned}
y_{t}(u)=m_{t-1-}^{1} & \delta^{1}\left(u+e_{1}\right) \\
+ & \left(\kappa_{t-\delta^{3}\left(u+e_{1}-e_{2}\right)}^{3}\left(\kappa^{3}\left(u+e_{1}-e_{2}\right)\right) .\right.
\end{aligned}
$$

By using the properties of the $\kappa$ and $\delta$ functions it follows that $y_{t}(u)$ is of the correct form and the proof of the induction step is finished.

The fact that destinations of sessions can successfully decode follows as a simple corollary to Lemma 1.

Corollary 1. At the end of time slot $t$ node $u_{1}$ can decode $m_{t+1-\delta^{3}\left(u_{1}\right)}^{3}\left(\kappa^{3}\left(u_{\mathrm{t}}\right)\right)$ as

$$
m_{t+1-\delta^{3}\left(u_{\mathrm{t}}\right)}^{3}\left(\kappa^{3}\left(u_{\mathrm{t}}\right)\right)=y_{t}\left(u_{\mathrm{t}}\right)+m_{t-1}^{1}\left(\kappa^{1}\left(u_{\mathrm{t}}\right)\right) .
$$

\section{Proof of Theorem 1, $\mathrm{P} / \mathrm{M}$ model}

From the construction in the previous subsections we have achieved a throughput of $1 / 7$ for $4 K$ sessions, the total number of hops of which is $3 K(K+1)$. The number of nodes in the network is $(K+2)^{2}$. Therefore the achieved number of bits $\times$ hops per time slot per node approaches $3 / 7$ in the limit of large $K$.

\section{Achievable Strategy under B/P}

The achievable strategy under the $B / P$ is similar to the strategy under $\mathrm{P} / \mathrm{M}$ and again based on [7]. The crucial element that needs to be addressed is that under the $\mathrm{B} / \mathrm{P}$ model sources and destinations will need to transmit twice, as is the case for the strategy considered in [7]. 


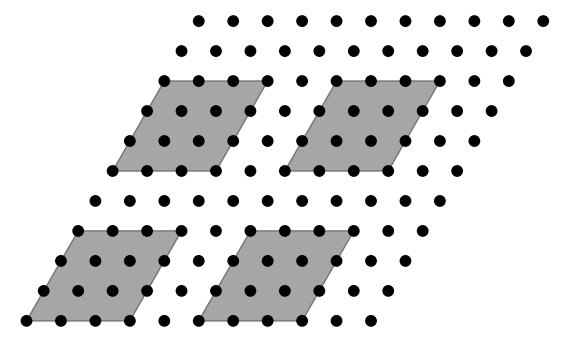

Fig. 4. The sets $S\left(\alpha_{h}, \alpha_{v}, 0\right)$, i.e., $\alpha_{o}=0$, for a value $L=2$.

We assume that the network is of size $(K+2) \times(K+2)$, where $K+2=(L+1)(L+3)$ for an integer $L$. The elements of the strategy are:

1) Construct subsets of the network of size $(L+2) \times(L+2)$.

The number of such subsets is $L^{3}$. This implies that some sets overlap.

2) In each subset of size $(L+2) \times(L+2)$ construct $4 L+1$ sessions in the same way as was done for the $\mathrm{P} / \mathrm{M}$ model on the whole $K \times K$ network, see Subsection IV-A.

3) Operate in rounds of $7 L+4$ time slots.

4) In each round deliver, for each session, one symbol at each receiver.

Again the different elements are addressed in subsequent subsections.

\section{A. Definition of subsets}

Let $S_{0}=\{u \in V \mid 0 \leq u[1] \leq L+1,0 \leq u[2] \leq L+1\}$. We consider $L^{3}$ different subsets of $V$, constructed from $S_{0}$ by using an offset. The new subsets are indexed by the three parameters $\alpha_{h}, \alpha_{v}, \alpha_{o}$, each chosen from the set $\{0,1, \ldots, L-$ $1\}$. The subsets are defined as

$$
\begin{aligned}
& S\left(\alpha_{h}, \alpha_{v}, \alpha_{o}\right)=S_{0}+\alpha_{h} e_{1}(L+3)+ \\
& \alpha_{v} e_{2}(L+3)+\alpha_{o}\left(e_{1}+e_{2}\right) .
\end{aligned}
$$

Some sets $S\left(\alpha_{h}, \alpha_{v}, 0\right)$, i.e., with $\alpha_{o}=0$, are depicted in Figure 4. Let $I_{0}, I\left(\alpha_{h}, \alpha_{v}, \alpha_{o}\right)$ and the other components of a subset be defined according to (1), w.r.t. the set $S_{0}$ and $S\left(\alpha_{h}, \alpha_{v}, \alpha_{o}\right)$ respectively.

The subsets have been defined in such a way, that even though each node is part of a number of subsets that grows with $L$, it is at the border of at most 4 such subsets.

\section{Lemma 2. For any $u \in V$}

$$
\left|\left\{\left(\alpha_{h}, \alpha_{v}, \alpha_{o}\right) \mid u \in C\left(\alpha_{h}, \alpha_{v}, \alpha_{o}\right)\right\}\right| \leq 4 .
$$

Proof: If $u \in L\left(\alpha_{h}, \alpha_{v}, \alpha_{o}\right)$, then there is no $\left(\tilde{\alpha}_{h}, \tilde{\alpha}_{v}, \tilde{\alpha}_{o}\right) \neq\left(\alpha_{h}, \alpha_{v}, \alpha_{o}\right)$ for which $u \in L\left(\tilde{\alpha}_{h}, \tilde{\alpha}_{v}, \tilde{\alpha}_{o}\right)$. The same holds for the other border types.

\section{B. Scheduling}

We use rounds of $7(L+4)$ time slots. The schedule consists of two parts. The first part assigns to each time slot a set of nodes that are scheduled in that time slot. The second part assigns to a scheduled node a set of parameters $\alpha_{h}, \alpha_{v}$ and $\alpha_{o}$.
In the $i$ th time slot of a round node $u$ transmits iff $u \in$ $A_{i}$, as defined in (4). The resulting schedule has the property that in each time slot, no node in the network has more than one scheduled neighbour. Also, in each round, each node is scheduled $L+4$ times.

Next, for each node $u$ we assign it's $K+4$ scheduled slots to triples $\left(\alpha_{h}, \alpha_{v}, \alpha_{o}\right)$. This is done by considering all triples $\left(\alpha_{h}, \alpha_{v}, \alpha_{o}\right)$. If $u \in I\left(\alpha_{h}, \alpha_{v}, \alpha_{o}\right)$ one slot is assigned to $\left(\alpha_{h}, \alpha_{v}, \alpha_{o}\right)$, if $u \in C\left(\alpha_{h}, \alpha_{v}, \alpha_{o}\right)$, two time slots are assigned to $\left(\alpha_{h}, \alpha_{v}, \alpha_{o}\right)$. By Lemma $2, K+4$ slots suffice.

The resulting scheduling is such that for each triple $\left(\alpha_{h}, \alpha_{v}, \alpha_{o}\right)$ we have:

1) All nodes in the interior of $S\left(\alpha_{h}, \alpha_{v}, \alpha_{o}\right)$ transmit once.

2) All nodes at the border of $S\left(\alpha_{h}, \alpha_{v}, \alpha_{o}\right)$ transmit twice.

3) All transmissions are successfully received by all neighbours.

4) No nodes in $S\left(\alpha_{h}, \alpha_{v}, \alpha_{o}\right)$ are receiving from nodes outside $S\left(\alpha_{h}, \alpha_{v}, \alpha_{o}\right)$.

\section{Coding}

Since, the scheduling defined above completely decouples the different subsets, we can apply the coding scheme from [7] to each of the subsets individually.

\section{Proof of Theorem 1, B/P model}

The achieved number of bit $\times$ hops per unit time is

$$
\frac{L^{3} 3 L(L+1)}{7(L+4)}=\frac{3}{7} L^{4}+\text { o }\left(L^{4}\right),
$$

which normalized by the network size of $L^{4}+o\left(L^{4}\right)$ is approaching $3 / 7$ in the limit of large $L$.

\section{ACKNOWLEDGEMENT}

Part of this work was done while J.H. Weber was visiting the Centre Interfacultaire Bernoulli (CIB) at EPFL in September/October 2011. The hospitality and support provided by CIB are greatly acknowledged. This work was supported in part by the European ERC Starting Grant 259530-ComCom and by NWO grant 612.001 .107 .

\section{REFERENCES}

[1] B. Nazer and M. Gastpar, "Compute-and-forward: Harnessing interference through structured codes," IEEE Trans. Inf. Theory, vol. 57, no. 10, pp. 6463-6486, 2011.

[2] J. Goseling, M. Gastpar, and J. H. Weber, "Line and lattice networks under deterministic interference models," IEEE Trans. Inf. Theory, vol. 57, no. 5, pp. 3080-3099, 2011

[3] S. Katti, H. Rahul, W. Hu, D. Katabi, M. Médard, and J. Crowcroft, "XORs in the air: practical wireless network coding," in Proc. of ACM SIGCOMM, 2006, pp. 243-254.

[4] S. Zhang, S. Liew, and P. Lam, "Hot topic: physical-layer network coding," in Proc. of the 12th Annual International Conference on Mobile Computing and Networking, 2006, pp. 358-365.

[5] S. Katti and D. Katabi, "Embracing wireless interference: Analog network coding," in Proc. of the 2007 Conference on Applications, Technologies, Architectures, and Protocols for computer communications, 2007, pp. 397-408.

[6] B. Nazer and M. Gastpar, "Reliable physical layer network coding," Proceedings of the IEEE, vol. 99, no. 3, pp. 438-460, 2011.

[7] J. Goseling, R. Matsumoto, T. Uyematsu, and J. H. Weber, "Lower bounds on the maximum energy benefit of network coding for wireless multiple unicast," Eurasip Journal on Wireless Communications and Networking, 2010 , special issue on network coding for wireless networks. 